\title{
Isolation of Nonpathogenic Mutants of Fusarium oxysporum f. sp. melonis for Biological Control of Fusarium Wilt in Cucurbits
}

\author{
Stanley Freeman, Aida Zveibil, Haim Vintal, and Marcel Maymon
}

Department of Plant Pathology, ARO, The Volcani Center, Bet Dagan 50250, Israel.

Accepted for publication 16 October 2001.

\section{ABSTRACT}

Freeman, S., Zveibil, A., Vintal, H., and Maymon, M. 2002. Isolation of nonpathogenic mutants of Fusarium oxysporum f. sp. melonis for biological control of Fusarium wilt in cucurbits. Phytopathology 92:164-168.

Two nonpathogenic mutant strains $4 / 4$ and $15 / 15$ of Fusarium oxysporum f. sp. melonis (race 1,2) were isolated by a continuous dipinoculation technique following UV mutagenesis of the virulent wildtype isolate FOM1.2. No disease symptoms or detrimental effects were observed following inoculation of muskmelon seedlings by strain $4 / 4$. In contrast, strain $15 / 15$ caused mortality of susceptible cultivars although to a lesser extent than the wild-type isolate. Strain $4 / 4$ colonized a variety of muskmelon and watermelon cultivars. In muskmelon cv. Ein Dor, seedlings were dipped in a conidial suspension of strain $4 / 4$ and planted in medium amended with the mutant to achieve $100 \%$ colonization of roots and between 30 to $70 \%$ of the lower stem tissues 7 days after planting. Similar percent colonization of watermelon seedlings by strain $4 / 4$ was recorded. In cross-protection experiments with muskmelon cultivars, significant reduction in seedling mortality was observed between 4/4colonized FOM1.2. challenged plants compared with that of wild-type challenged plants alone. Similarly, strain $4 / 4$ was able to significantly reduce mortality of watermelon seedlings caused by $F$. oxysporum $\mathrm{f}$. sp. niveum race 2 . This novel approach of generating nonpathogenic mutants for biological control in Fusarium spp. and other fungal pathogens from virulent wild-type isolates may be beneficial for control, because the mutant strains, lacking only in pathogenicity, may compete more efficiently than other biocontrol organisms against the pathogen of origin.
Fusarium wilt of muskmelon and watermelon caused by $F u$ sarium oxysporum f. sp. melonis and $F$. oxysporum f. sp. niveum, respectively, inflicts considerable yield loss worldwide $(7,17)$. Due to the persistent nature of these pathogens in soil, once the disease is established in the field, the pathogen is likely to remain indefinitely because subsequent crops of susceptible melon and watermelon cultivars increase pathogenic populations. Fusarium spp. of muskmelon and watermelon cause plant wilting by colonizing the hosts vascular system, eventually resulting in seedling or mature plant mortality. Due to the persistent nature of these wilt pathogens, the diseases are best managed with wilt-resistant cultivars. However, as resistant cultivars are utilized, new virulent populations (physiological races) may develop in specific locations. In $F$. oxysporum f. sp. melonis, four common races exist worldwide and have been designated $0,1,2$, and 1,2 $(9,15,23,29)$. Race 1,2 is virulent to muskmelon cultivars possessing resistance genes FOM-1 and FOM-2 $(17,29)$ and, to date, no known field resistant cultivars are commercially available to this pathogen. With F. oxysporum f. sp. niveum, a similar phenomenon of resistance is found. Whereas resistant watermelon cultivars have been developed to races 0 and 1 , no commercially resistant cultivars are currently available to race $2(7,16,26)$.

The wide use of synthetic chemicals has caused the development of resistant pathogenic populations. Resistance to biocontrol microorganisms of fungal pathogens has not been reported and is considered an environmental-friendly approach. The use of saprophytic or nonpathogenic isolates of Fusarium spp. for biological control of pathogenic Fusarium spp. in various crops has been extensively studied and applied $(2,21,24,30,33)$. Likewise, certain pathogenic races of Fusarium spp. are able to protect plants from specific pathogens $(7,19)$. The proposed mechanisms in-

Corresponding author: S. Freeman; E-mail address: freeman@ netvision.net.il

Publication no. P-2001-1205-02R

(C) 2002 The American Phytopathological Society volved in Fusarium spp. biological control include (i) competition for infection sites $(1,5,24,30)$, (ii) competition for nutrients $(1,2$, $11,20)$, and (iii) local and systemic induced resistance $(5,7,19$, 20,24,33).

In an effort to introduce new biocontrol approaches, we have attempted to develop biocontrol relying on nonpathogenic mutant strains derived from the wild-type pathogens themselves. It was previously demonstrated that UV mutagenesis of a wild-type pathogen of Colletotrichum magna could result in genetic conversion to a nonpathogenic, endophytic mutualist (14). The nonpathogenic mutant strain (path-1) of C. magna retained the wild-type phenotype, colonized cucurbit and other host tissues, and protected plants against the wild type and $F$. oxysporum $\mathrm{f}$. sp. niveum in watermelon by priming the host defense response $(14,27)$. In view of the ability to generate a mutualist from a pathogen in Colletotrichum spp., the main objectives of this study were to (i) isolate nonpathogenic mutants of $F$. oxysporum f. sp. melonis, and (ii) test the feasibility of these mutants in protecting different cucurbit cultivars against their respective Fusarium wilt pathogens. Although the C. magna mutant was efficacious in disease control, the transfer of an isolate from one country to another and distribution in the field can be limiting factors for biological control; therefore, generation of endemic Fusarium mutants in specific environments may alleviate these problems.

\section{MATERIALS AND METHODS}

Fungal isolates and inocula production. Pathogenic wild-type isolates of $F$. oxysporum f. sp. melonis (FOM1.2, race 1,2) and $F$. oxysporum f. sp. niveum (FON2, race 2) used in this study were isolated from infected muskmelon and watermelon, respectively $(17,26)$. Conidial suspensions of the wild type and nonpathogenic mutant strains were grown on a medium containing Difco (Becton Dickinson Microbiology Systems, Sparks, MD) yeast extract (5 g/liter), Difco bactopeptone (5 g/liter), glucose (20 g/liter), and Difco agar (12 g/liter) in petri plates and incubated for 5 days at 
$23^{\circ} \mathrm{C}$ under cool light fluorescent illumination for routine culturing. Conidia for inoculation purposes were obtained by placing 10 hyphal disks (5-mm diameter) from the margins of 5-day-old fungal colonies into a $250-\mathrm{ml}$ flask containing $50 \mathrm{ml}$ of FLC medium (8) on a rotary shaker at $150 \mathrm{rpm}$ for 4 to 5 days. The ingredients of the FLC medium were as follows: $0.25 \mathrm{~g}$ of $\mathrm{MgSO}_{4}$, $1.5 \mathrm{~g}$ of $\mathrm{KH}_{2} \mathrm{PO}_{4}, 2 \mathrm{~g}$ of $\mathrm{KNO}_{3}, 10 \mathrm{~g}$ of Difco yeast extract, and $20 \mathrm{~g}$ of sucrose per 1 liter of distilled water, which was adjusted to $\mathrm{pH}$ 5.5. The medium was autoclaved and trace element solution consisting of $0.865 \mathrm{~g}$ of $\mathrm{Na}_{2} \mathrm{~B}_{4} \mathrm{O}_{7} \cdot 10 \mathrm{H}_{2} \mathrm{O}, 4.82 \mathrm{~g}$ of $\mathrm{FeCl}_{2} \cdot 6 \mathrm{H}_{2} \mathrm{O}$, $0.129 \mathrm{~g}$ of $\left(\mathrm{NH}_{4}\right)_{6} \mathrm{Mo}_{4} \mathrm{O}_{24} \cdot 4 \mathrm{H}_{2} \mathrm{O}, 0.378 \mathrm{~g}$ of $\mathrm{Cu}\left(\mathrm{NO}_{3}\right)_{2} \cdot 3 \mathrm{H}_{2} \mathrm{O}$, and $4.43 \mathrm{~g}$ of $\mathrm{ZnSO}_{4} \cdot 7 \mathrm{H}_{2} \mathrm{O}$ per liter was added later $(1 \mathrm{ml}$ of trace element solution per 1 liter of FLC medium). After 5 days growth in FLC medium, the contents were filtered through eight layers of surgical gauze and centrifuged at $8,000 \times g$ for $10 \mathrm{~min}$. The conidial pellets were rinsed once in sterile distilled water and adjusted with $0.05 \%$ agar to a concentration of $1.5 \times 10^{6}$ or $2 \times$ $10^{7}$ conidia per $\mathrm{ml}$ for inoculation purposes.

Plants. Cultivars of muskmelon (cvs. Ein Dor and Yokneam, susceptible to races $0,1,2$, and 1,2 of F. oxysporum f. sp. melonis, and the partially resistant differential $\mathrm{cv}$. Hemed, susceptible to races 0,1 , and 1,2 of $F$. oxysporum $\mathrm{f}$. sp. melonis) and watermelon (cvs. Malali and Odem, susceptible to races 0,1 , and 2 of $F$. oxysporum f. sp. niveum) used in this study were received from Zeraim Gedera Seed Company, Gedera, Israel.

Mutagenesis. Conidia of $F$. oxysporum f. sp. melonis (isolate FOM1.2) were adjusted to a concentration of $10^{8}$ per milliliter without washing, and aliquots of $5 \mathrm{ml}$ were dispensed aseptically in glass petri plates. Plates containing the conidia were placed at a distance of $5 \mathrm{~cm}$, directly under UV light $(3 \times 15 \mathrm{~W}, 254-\mathrm{nm}$ tubes, $90 \mathrm{~W}$; Vilber Laumat, Marne La Vallee, France) and exposed for 2 to $3 \mathrm{~min}$. These exposure rates resulted in mortality of approximately $99.99 \%$ of the conidia, determined by dilution plating on potato dextrose agar. Surviving individual colonies were screened for pathogenicity on Fusarium-susceptible muskmelon cultivars.

Screening surviving isolates after UV irradiation for pathogenicity by a continuous dip method. The procedure used in this study was similar to that previously described (12). Muskmelon seedlings of cvs. Ein Dor and Hemed were germinated in a vermiculite-rooting medium. After 5 to 6 days, seedlings were removed and the roots were washed under running tap water. Seedlings were exposed continuously in $18-\mathrm{ml}$ conidial suspensions of the UV-irradiated strains (approximately $10^{6}$ conidia per $\mathrm{ml}$ of $0.05 \%$ agar) in standard scintillation vials of 20 -ml capacity (10 plants per vial). The plants were maintained under controlled conditions at $24 \pm 2{ }^{\circ} \mathrm{C}$ with a 12-h diurnal irradiation fluorescent light cycle. Disease symptoms were typified by cotyledon wilting and yellowing, accompanied by lesions along the stems. Potential nonpathogenic mutants were isolated by this method and further verified by a root-dip inoculation method.

Further screening of potential nonpathogenic mutants in pots by the standard root-dip method. The standard root-dip inoculation method used was essentially as previously described $(13,22)$ to verify the results obtained by the continuous dip-inoculation technique. 'Ein Dor' and 'Hemed' seedlings were germinated in vermiculite, removed and washed after 5 to 6 days, and dipped for $30 \mathrm{~s}$ in a conidial suspension $\left(10^{6}\right.$ conidia per $\left.\mathrm{ml}\right)$ of the wild-type isolate FOM1.2, and the potential nonpathogenic mutants were prescreened as nonpathogens by the continuous dipinoculation assay. Thereafter, seedlings were planted in pots $(10 \times$ $10 \times 12 \mathrm{~cm}$ tall) in a peat/vermiculite mixture $(1: 2, \mathrm{vol} / \mathrm{vol})$. Disease incidence was observed after 6 to 7 days and expressed by the number of diseased seedlings as a percentage of the total number inoculated and compared with that of uninoculated controls. The screening experiments consisted of five replicates of 10 seedlings per treatment, arranged in a completely randomized design, and were conducted at least five times.
Plant colonization assays. Seedlings of muskmelon cvs. Ein Dor and Yokneam and watermelon cvs. Odem and Malali were tested for colonization by a nonpathogenic mutant strain $4 / 4$ derived from isolate FOM1.2. The seedlings were germinated in vermiculite medium, dipped for $30 \mathrm{~min}$ in a conidial suspension of strain $4 / 4\left(1.5 \times 10^{6}\right.$ conidia per $\left.\mathrm{ml}\right)$, and transferred to a peat/ vermiculite rooting mixture $(1: 2, \mathrm{vol} / \mathrm{vol})$ containing $2 \times 10^{7}$ conidia per gram of mixture. Plants were sampled at 2, 4, 7, and 10 days after transfer to the nonpathogenic mutant-amended growth medium to determine percent colonization of roots, crown, and lower and upper stem sections. The plant sections were surface sterilized by submerging in 3\% sodium hypochlorite for $2 \mathrm{~min}$, washing in sterilized distilled $\mathrm{H}_{2} \mathrm{O}$ for $1 \mathrm{~min}$, and drying in a laminar flow hood. Each section was then divided into five separate subsections and colonization was assessed by plating on a Fusarium-selective medium (18). Noninoculated seedlings were used as a negative control to assess whether other Fusarium populations were present and if they colonized seedlings. Five plants of each cultivar were sampled at each period, and the experiments, arranged in a completely randomized design, were conducted twice for each cultivar.

Plant inoculation assays. The nonpathogenic mutant strains were assessed for pathogenicity and control of the wild-type isolates FOM1.2 in muskmelon and FON2 in watermelon. Seedlings of muskmelon cv. Ein Dor and watermelon cv. Odem were colonized by the mutant strain $4 / 4$ as previously described, or maintained in noncolonized rooting medium to serve as wild-type inoculated controls. The seedlings were grown for 7 days in either nonamended medium before wild-type inoculation or in 4/4amended medium to allow maximum colonization by strain $4 / 4$ before challenge inoculation with a $20-\mathrm{ml}$ conidial suspension of $1.5 \times 10^{6}$ conidia per $\mathrm{ml}$ of the respective wild-type isolates FOM1.2 and FON2. Disease incidence was observed 6 to 7 days after inoculation, monitored periodically, and expressed as the percentage of seedling mortality compared with that of noninoculated water controls. Experiments consisted of five replicates with 10 seedlings per treatment arranged in a completely randomized design. Each biocontrol experiment was conducted at least five times under controlled conditions for each cucurbit cultivar as described previously.

Statistical analyses. Analysis of variance (ANOVA) indicated that the interaction of experiments-treatments (different isolates and effect of strain 4/4 on biological control) was insignificant $(P=0.05)$, therefore data of the plant inoculation experiments were pooled before analyses. The period used for calculating the area under the disease progress curve (AUDPC) extended from the date of inoculation to the last disease assessment. AUDPC units are proportion-days. For comparing AUDPC values from different experiments, the relative area under the disease progress curve (RAUDPC) was calculated by dividing the AUDPC value by the duration of the epidemic and multiplying the outcome by 100 . RAUDPC units are percentages. Statistical analyses of the data were conducted using the JMP software package, version 3.2.6 (SAS Institute Inc., Cary, NC) at a significance level of $P=0.05$.

\section{RESULTS}

Isolation of nonpathogenic mutants from $F$. oxysporum $\mathrm{f}$. sp. melonis. Approximately 800 strains derived from wild-type race 1,2 of $F$. oxysporum f. sp. melonis that survived the UV irradiation were tested for pathogenicity by the continuous dip-inoculation screening method. Of these survivors, two strains were further tested by the standard root-dip method (Fig. 1). Strain $4 / 4$ remained completely nonpathogenic after inoculating both susceptible Ein Dor and partially resistant Hemed muskmelon cultivars. No stunting, chlorosis, or any other detrimental effects were observed in strain 4/4-inoculated seedlings compared with noninoculated water controls. We have been working with strain $4 / 4$ for the 
past 7 years and the nonpathogenic trait of this strain has remained stable without any record of reversion to its original pathogenic state. Strain $4 / 4$ could be easily identified in culture according to its light pink pigmentation compared with dark purple of the wild type. In contrast to strain 4/4, strain $15 / 15$ caused mortality of $\mathrm{cv}$. Ein Dor seedlings (Fig. 1) but was nonpathogenic on differential cv. Hemed. RAUDPC for inoculated cv. Ein Dor seedlings with isolates FOM1.2, 15/15, and 4/4 were 73.1 $\pm 1.3,43.2 \pm 3.8$, and 0 , respectively. Comparing the RAUDPC values of the three isolates with a one-way ANOVA revealed that all treatments were significantly different from each other ( $F$ ratio of $257.0 ; P \leq$ 0.0001 ). Because strain $15 / 15$ retained a measure of pathogenicity when inoculating susceptible cv. Ein Dor, further experiments utilizing this mutant for biological control were not pursued extensively.

Seedling colonization by nonpathogenic mutant strain $\mathbf{4 / 4}$. The extent of colonization of muskmelon and watermelon seedlings by the mutant strain 4/4 was examined after planting in artificially inoculated growth medium. In both muskmelon and watermelon cvs. Ein Dor and Odem, respectively, strain 4/4 was isolated from upper stem sections (Fig. 2). In muskmelon, root colonization reached $100 \% 7$ days after planting, while crown and lower and upper stem were also colonized to certain degrees (Fig. 2A), increasing according to exposure time to the inoculum. Similar colonization percentages were recorded in watermelon seedlings of cv. Odem 10 days after planting in 4/4-inoculated growth medium (Fig. 2B), although initial root and crown colonization progressed more rapidly in watermelon than in muskmelon (Fig. 2). Similar colonization results were determined for muskmelon cv. Yokneam and watermelon cv. Malali (data not shown). No colonization by Fusarium spp. was detected in noninoculated water control seedlings.

Biological control of wild-type isolates FOM1.2 and FON2 by nonpathogenic mutant strain $\mathbf{4 / 4}$. Cucurbit seedlings precolonized by mutant strain $4 / 4$ were assessed for protection against the wild-type isolates FOM1.2 in muskmelon and FON2

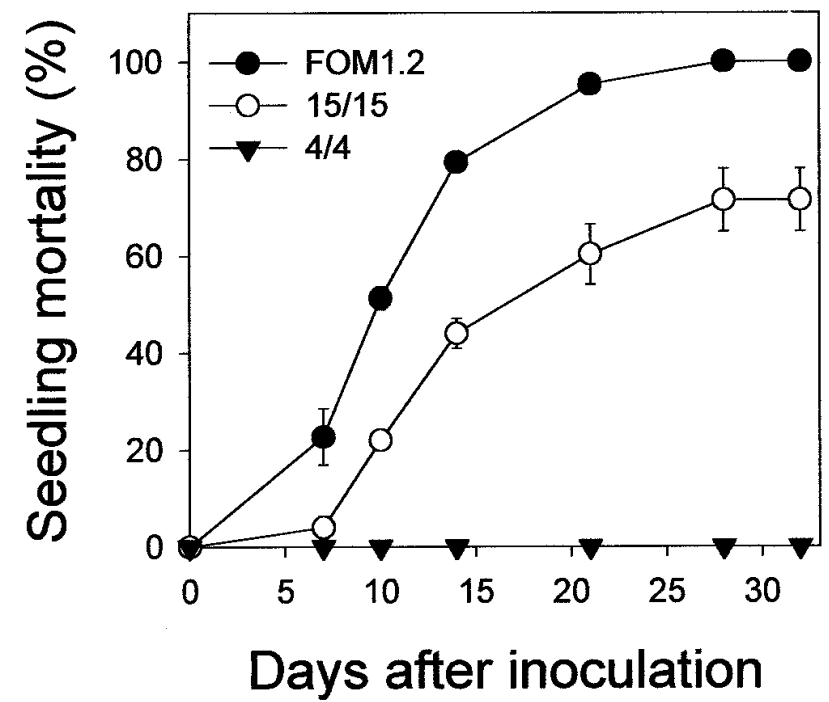

Fig. 1. Seedling mortality in muskmelon cv. Ein Dor inoculated with nonpathogenic strains $15 / 15$ and 4/4 and the wild-type isolate FOM1.2 of Fusarium oxysporum f. sp. melonis. Seedlings were assayed by the standard root-dip procedure by immersing roots for $30 \mathrm{~s}$ in conidial suspensions $\left(10^{6}\right.$ conidia per $\mathrm{ml}$ ) and transplanting into noninfested potting mixture. Relative area under the disease progress curve (RAUDPC) for isolates FOM1.2, 15/15, and $4 / 4$ were $73.1 \pm 1.3,43.2 \pm 3.8$, and 0 , respectively. Comparing the RAUDPC values of the three isolates with a one-way analysis of variance revealed that all treatments were significantly different from each other ( $F$ ratio of 257.0; $P \leq 0.0001)$. Bars represent standard error of the means for each value. Where bars are missing, the error is less than $5 \%$ of the value of the point. No disease was observed in noninoculated water control seedlings. in watermelon (Fig. 3). In all the experiments, strain 4/4 did not have any adverse effects on the seedlings and neither disease symptoms nor mortality were observed. In both the respective muskmelon and watermelon cultivars, strain 4/4 consistently reduced seedling mortality caused by FOM1.2 (Fig. 3A) and FON2 (Fig. 3B). In muskmelon, RAUDPC values for isolates FOM1.2, 4/4-inoculated challenged with FOM1.2 and 4/4 alone were 70.9 \pm $1.8,26.5 \pm 7.6$, and 0 , respectively. All treatments were significantly different from each other when comparing the RAUDPC values by one-way ANOVA ( $F$ ratio of $322.0 ; P \leq 0.0001$ ) (Fig. 3A).

Similar results were obtained in watermelon (Fig. 3B). Seedling mortality was reduced in 4/4-precolonized plants following challenge inoculation by isolate FON2 compared with that of isolate FON2-inoculated seedlings alone. RAUDPC values for isolates FON2, 4/4-inoculated challenged with isolate FON2, and 4/4 alone were $54.0 \pm 2.9,23.2 \pm 5.8$, and 0 , respectively. All treatments were significantly different from each other when comparing the RAUDPC values by one-way ANOVA ( $F$ ratio of 53.0; $P \leq 0.0001)$ (Fig. 3B).

\section{DISCUSSION}

In this study, we have isolated a nonpathogenic mutant fungus 4/4 derived from the wild-type $F$. oxysporum f. sp. melonis following UV mutagenesis by a continuous dip-inoculation method. Mutant 4/4 colonized a variety of muskmelon and watermelon cultivars without causing any disease symptoms. In cross-protection experiments with melon (cv. Ein Dor) and watermelon (cv. Odem) cultivars, significant disease reduction was achieved when comparing 4/4-colonized wild-type-challenged seedlings with those of wild-type-challenged alone. Initially, the continuous dipinoculation method was devised as a rapid, reliable bioassay for determining pathogenicity in $C$. magna and for large-scale screening and isolation of nonpathogenic mutants in this pathogen (12). This method was also reliable and consistent for pathogenicity determination of various Fusarium isolates in whole plant assays of watermelon and muskmelon, and disease development corresponded well to that of the standard pot-inoculation procedure for whole plants (13).

The plant-pathogen interaction of Fusarium spp. with its various hosts has been extensively studied. Initially, Fusarium pathogens penetrate the vasculature via root tips. Thereafter, in tomato for example, vascular colonization by $F$. oxysporum f. sp. lycopersici is extensive in wilt-susceptible plants but remains limited to the basal part of resistant plants (6). It is presumed that resistant tomato plants are able to "wall-off" potential $F$. oxysporum f. sp. lycopersici isolates by trapping conidia, depositing callose, tylose occlusion, infusing phenolic compounds, and synthesizing phytoalexins (6). The pathogen may cause disease by avoiding or overcoming any of these defense mechanisms or by vascular occlusion. The cause of wilting by the pathogen may be due to either vascular occlusion resulting in the failure of water transport or toxin production. With $F$. oxysporum f. sp. melonis however, wilt-resistant muskmelon plants also harbor the pathogen extensively within the vascular elements $(10,25)$. This phenomenon may be due to degradation of fungal cell wall-degrading enzymes (CWDEs) by the resistant host as a defense response or alternatively, the fungus may not be expressing these factors in resistant plants, but the mechanisms governing this resistance are unknown. Likewise, the resistance mechanisms in watermelon have not been elucidated (8).

CWDEs are known factors contributing to fungal pathogenicity $(3,4)$. Although CWDEs have been isolated and characterized from a number of pathogenic fungi, the role of these enzymes in plant pathogenesis is not well understood. Most reports have shown that purified pectinases are capable of reproducing disease symptoms, that there is a connection between amount, timing, and type of enzyme production to that of disease symptoms, and in 
certain host-pathogen interactions, a lack of pectate lyase and polygalacturonase activities cause reduced to no pathogenicity $(3,4,32)$. Accordingly, it was shown that a nonpathogenic mutant of C. magna (path-1) colonized and proliferated within host tissue without causing disease symptoms (14). Colonized watermelon plants were protected between 90 to $100 \%$ against disease caused by $C$. orbiculare (28). Biochemical studies indicated that path-1 alone avoided activation of host defenses, but path-1-colonized plants challenged with virulent isolates activated host defenses more rapidly than control plants (28). In contrast, however, path-1 alone was capable of inducing resistance against the postharvest pathogen $C$. gloeosporioides in avocado fruit by increasing levels of an antifungal diene in pretreated fruit (27). This mutant was reported to lack extracellular pectate lyase and possess reduced polygalacturonase activities and appeared to be deficient in extracellular secretion of these enzymes (31).

Preliminary work has been initiated regarding the mechanisms involved in elucidating the nonpathogenic traits of strain 4/4. It has been shown that there is a reduction in cellulytic activity of mutant 4/4 compared with that of the wild-type isolate FOM1.2, however, the amount, timing, and nature of enzymatic production needs to be determined (unpublished data). The mechanisms of

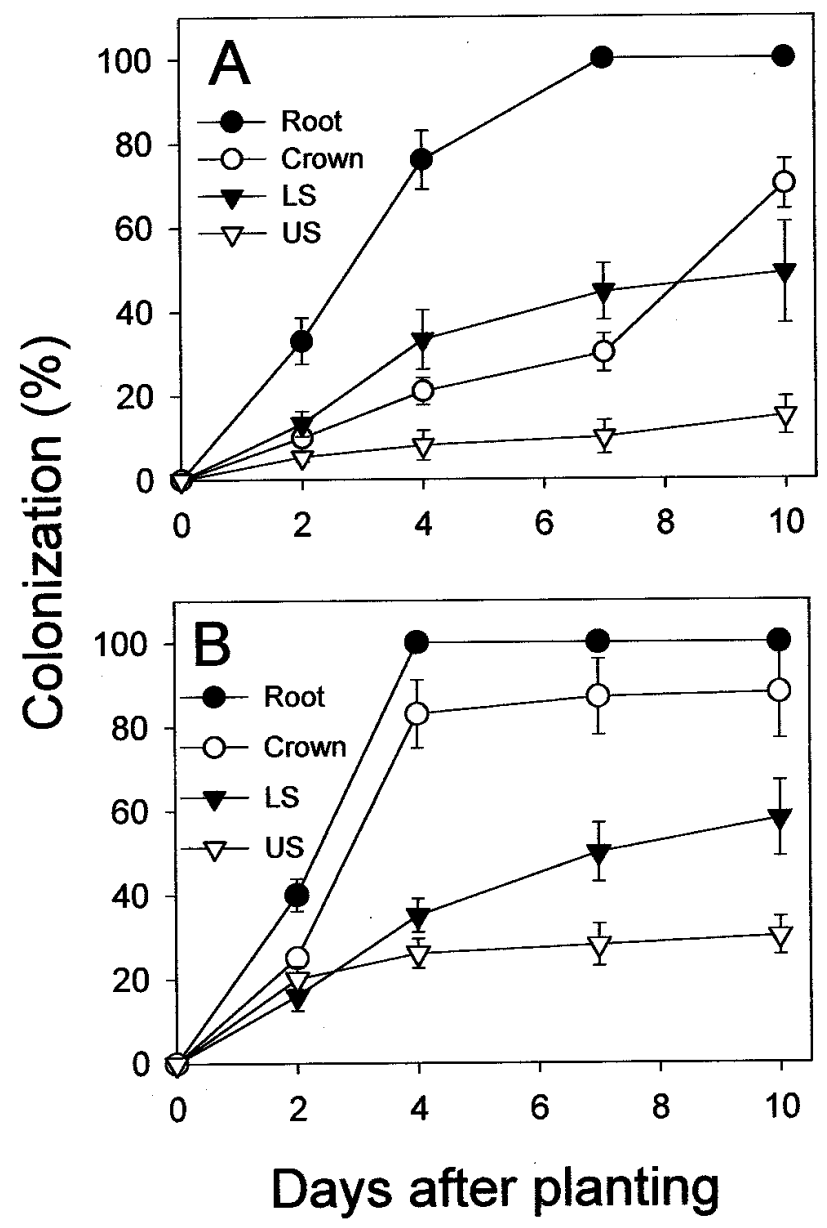

Fig. 2. Seedling colonization of A, muskmelon cv. Ein Dor and B, watermelon cv. Odem by nonpathogenic mutant strain 4/4 of Fusarium oxysporum f. sp. melonis. The seedlings were transferred to a rooting medium containing $10^{6}$ conidia per gram. Five plants were sampled at 2, 4, 7, and 10 days after transfer to the 4/4-amended growth medium to determine percent colonization of roots, crown, and lower (LS) and upper stem (US) sections by plating on a Fusarium-selective medium. Bars represent standard error of the mean calculated from pooled variance at each sampling point. Where bars are missing, the error is less than $5 \%$ of the value of the point. No colonization of Fusarium spp. was detected in seedlings grown in noninoculated growth medium that were used as negative controls. protection afforded by mutant strain $4 / 4$ have not been studied in depth, however, direct parasitism, hyphal interference, and toxin production against wild-type isolates FOM1.2 and FON2 were not observed (unpublished data), indicating that competition for nutrients and infection site and induced resistance may be alternative modes of action. Although disease reduction is decreased by strain $4 / 4$, it is not clear whether the wild-type isolates were prevented entry into the vasculature by the precolonization of strain $4 / 4$. Because 40 to $50 \%$ disease was recorded in 4/4-protected seedlings, it would appear that the wild-type isolates were able to penetrate and colonize these seedlings. However, once reporter genes are inserted in both wild-type and mutant isolates (work in progress), the interactions between these microorganisms in planta may be elucidated by these marked strains. Although only limited disease reduction was afforded by strain $4 / 4$, the potential for using this mutant or other strains and methods of control in combination should be assessed for improving disease reduction. It should be noted though that the experiments conducted in this study were carried out under controlled conditions with an inoculum density that may be much higher than that occurring in the field; therefore, the mutant strains may perform better under natural conditions with lower inoculum levels.

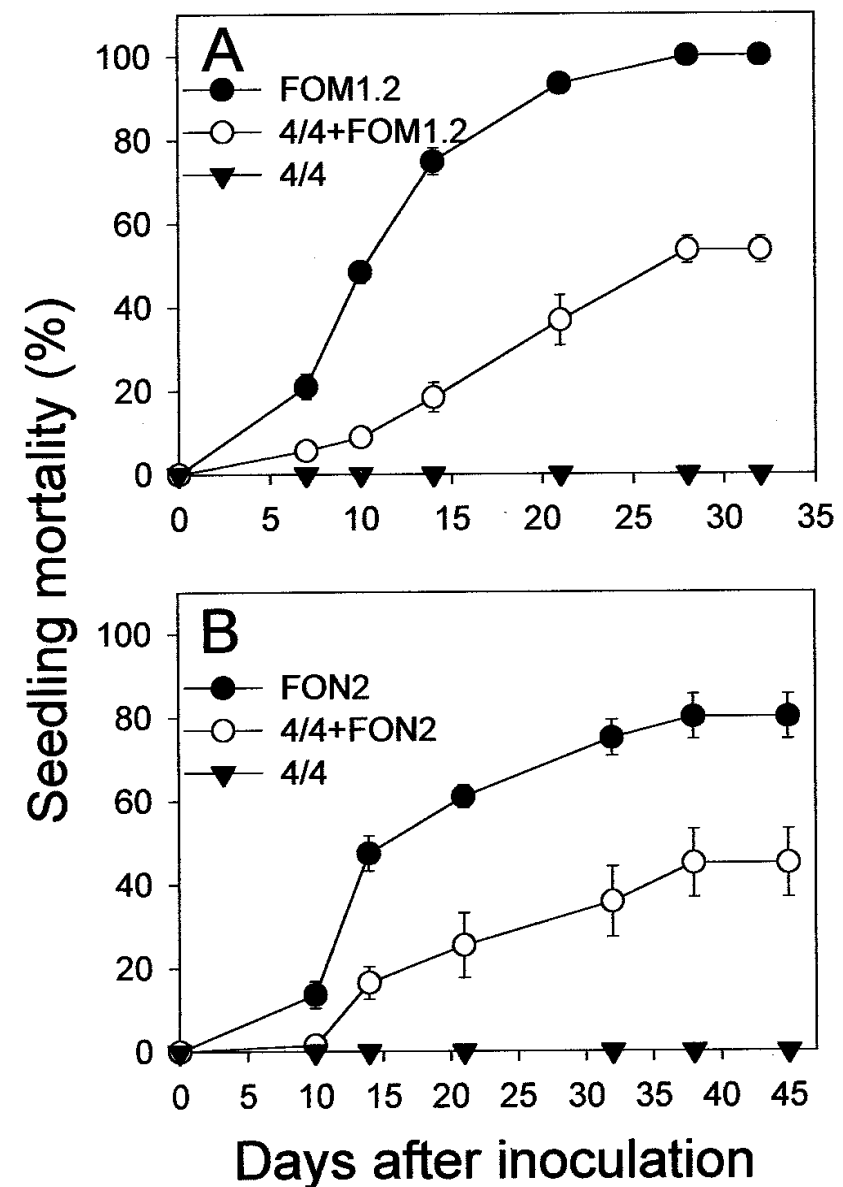

Fig. 3. Seedling mortality in A, muskmelon cv. Ein Dor and B, watermelon cv. Odem inoculated with the respective wild-type pathogenic isolates FOM1.2 of Fusarium oxysporum $\mathrm{f}$. sp. melonis and FON2 of F. oxysporum $\mathrm{f}$. sp. niveum, respectively, nonpathogenic mutant strain 4/4-colonized and wild-type challenge, and strain $4 / 4$ alone. The seedlings were grown for 7 days in either nonamended medium before wild-type inoculation or in 4/4amended medium, before challenge inoculation with 20 -ml conidial suspension of $1.5 \times 10^{6}$ conidia per $\mathrm{ml}$ of the respective wild-type FOM1.2 and FON2 isolates. In $\mathbf{A}$, muskmelon and $\mathbf{B}$, watermelon, relative area under the disease progress curve for all treatments was significantly different. Bars represent standard error of the means for each value. Where bars are missing, the error is less than $5 \%$ of the value of the point. No disease was observed in noninoculated water control seedlings. 
The nonpathogenic Fusarium and Colletotrichum mutants may serve as potential novel biocontrol agents. To the best of our knowledge to date, no biocontrol agents of Fusarium have been used that were derived from wild-type pathogenic isolates of Fusarium spp. For example, in Fusarium spp., only saprophytes and specific pathogenic isolates of this genus have been utilized for biological control. The nonpathogenic mutants therefore may compete better than their original pathotype when applied in the correct manner. This novel approach of generating nonpathogenic mutants may be used to improve biocontrol agents by allowing the mutants to compete more efficiently for infection sites and nutrient sources. Further research using these mutants may provide additional information toward a better understanding of host-parasite interactions, specifically, endophytes, pathogens, and their hosts. Besides the potential use as biocontrol agents, the nonpathogenic mutants will enable a better understanding of the genetic factors involved in pathogenicity. Complementation of nonpathogenic mutant strains, path-1 of C. magna (14), and 4/4 of F. oxysporum f. sp. melonis with wild-type genomic libraries may enable characterization of genes involved in pathogenicity.

\section{ACKNOWLEDGMENTS}

This research was supported in part by research grant IS-2520-95 from BARD, The United States-Israel Binational Agricultural Research and Development Fund, and grant 132-0922 from the Chief Scientist of the Israeli Ministry of Agriculture awarded to S. Freeman. We thank D. Shtienberg for assistance with statistical analyses.

\section{LITERATURE CITED}

1. Alabouvette, C. 1990. Biological control of Fusarium wilt pathogens in suppressive soils. Pages 27-43 in: Biological Control of Soilborne Plant Pathogens. D. Hornby, ed. CAB International, Wallingford, UK.

2. Alabouvette, C., and Couteaudier, Y. 1992. Biological control of Fusarium wilts with nonpathogenic Fusaria. Pages 415-426 in: Biological Control of Plant Diseases. E. C. Tjamos, G. C. Papavizas, and R. J. Cook, eds. Plenum Press, New York.

3. Alghisi, P., and Favaron, F. 1995. Pectin degrading enzymes and plantparasitic interactions. Eur. J. Plant Pathol. 101:365-375.

4. Annis, S. L., and Goodwin, P. H. 1997. Recent advances in the molecular genetics of plant cell wall-degrading enzymes produced by plant pathogenic fungi. Eur. J. Plant Pathol. 103:1-14.

5. Bao, J. R., and Lazarovits, G. 2001. Differential colonization of tomato roots by nonpathogenic and pathogenic Fusarium oxysporum may influence Fusarium wilt control. Phytopathology 91:449-456.

6. Beckman, C. H. 1987. The Nature of Wilt Diseases of Plants. The American Phytopathological Society, St. Paul, MN.

7. Biles, C. L., and Martyn, R. D. 1989. Local and systemic resistance induced in watermelons by formae speciales of Fusarium oxysporum. Phytopathology 79:856-860.

8. Biles, C. L., Martyn, R. D., and Netzer, D. 1990. In vitro inhibitory activity of xylem exudates from cucurbits towards Fusarium oxysporum microconidia. Phytoparasitica 18:41-49.

9. Cohen, R., Katan, T., Katan, J., and Cohn, R. 1989. Occurrence of Fusarium oxysporum $\mathrm{f}$. sp. melonis race 1,2 on muskmelon in Israel. Phytoparasitica 17:319-322.

10. Cohen, R., Riov, J., Lisker, N., and Katan, J. 1986. Involvement of ethylene in herbicide-induced resistance to Fusarium oxysporum f. sp. melonis. Phytopathology 76:1281-1285.

11. Couteaudier, Y. 1992. Competition for carbon in soil and rhizosphere, a mechanism involved in biological control of Fusarium wilts. Pages 99104 in: Biological Control of Plant Diseases. E. C. Tjamos, G. C. Papavizas, and R. J. Cook, eds. Plenum Press, New York.

12. Freeman, S., and Rodriguez, R. J. 1992. A rapid, reliable bioassay for pathogenicity of Colletotrichum magna on cucurbits, and its use for the isolation of nonpathogenic mutants. Plant Dis. 76:901-905.

13. Freeman, S., and Rodriguez, R. J. 1993. A rapid inoculation technique for assessing pathogenicity of Fusarium oxysporum f. sp. niveum and $F$. o. melonis on cucurbits. Plant Dis. 77:1198-1201.

14. Freeman, S., and Rodriguez, R. J. 1993. Genetic conversion of a fungal plant pathogen to an nonpathogenic, endophytic mutualist. Science 260: 75-78.

15. Gerlach, M., and Blok, W. J. 1988. Fusarium oxysporum f. sp. cucurbitacearum embracing all formae speciales of $F$. oxysporum attacking Cucurbitaceae. Neth. J. Plant Pathol. 94:17-31.

16. Hopkins, D. L., Lobinske, R. J., and Larkin, R. P. 1992. Selection for Fusarium oxysporum f. sp. niveum race 2 in monocultures of watermelon cultivars resistant to Fusarium wilt. Phytopathology 82:290-293.

17. Katan, T., Katan, J., Gordon, T. R., and Pozniak, D. 1994. Physiologic races and vegetative compatibility groups of Fusarium oxysporum f. sp. melonis in Israel. Phytopathology 84:153-157.

18. Komada, H. 1975. Development of a selective medium for quantitative isolation of Fusarium oxysporum from natural soil. Rev. Plant Prot. Res. 8:115-125.

19. Kroon, B. A. M., Scheffer, R. J., and Elgersma, D. M. 1991. Induced resistance in tomato plants against Fusarium wilt invoked by Fusarium oxysporum f. sp. dianthi. Neth. J. Plant Pathol. 97:401-408.

20. Larkin, R. P., and Fravel, D. R. 1999. Mechanisms of action and doseresponse relationships governing biological control of Fusarium wilt of tomato by nonpathogenic Fusarium spp. Phytopathology 89:1152-1161.

21. Larkin, R. P., Hopkins, D. L., and Martin, F. N. 1996. Suppression of Fusarium wilt of watermelon by nonpathogenic Fusarium oxysporum and other microorganisms recovered from a disease-suppressive soil. Phytopathology 86:812-819.

22. Latin, R. X., and Snell, S. J. 1986. Comparison of methods for inoculation of muskmelon with Fusarium oxysporum f. sp. melonis. Plant Dis. 70:297-300.

23. Leary, J. V., and Wilbur, W. D. 1976. Identification of the races of $F u$ sarium oxysporum f. sp. melonis causing wilt of muskmelon in California. Phytopathology 66:15-16.

24. Mandeel, Q., and Baker, R. 1991. Mechanisms involved in biological control of Fusarium wilt of cucumber with strains of nonpathogenic Fusarium oxysporum. Phytopathology 81:462-469.

25. Netzer, D., Kritzman, G., and Chet, I. 1979. $\beta$-(1,3) Glucanase activity and quantity of fungus in relation to Fusarium wilt in resistant and susceptible near-isogenic lines of muskmelon. Physiol. Plant Pathol. 14:4755 .

26. Netzer, D., and Weintal, C. 1989. Race 2 of Fusarium oxysporum f. sp. melonis new to Israel. Plant Dis. 64:853-854.

27. Prusky, D., Freeman, S., Rodriguez, R. J., and Keen, N. T. 1994. A nonpathogenic mutant strain of Colletotrichum magna induces resistance in avocado fruits to $C$. gloeosporioides. Mol. Plant-Microbe Interact. 7:326-333.

28. Redman, R. S., Freeman, S., Clifton, D. R., Morrel, J., Brown, G. S., and Rodriguez, R. J. 1999. Biochemical analysis of plant protection afforded by a non-pathogenic endophytic mutant of Colletotrichum magna (teleomorph: Glomerella magna; Jenkins and Winstead, 1964). Plant Physiol. 119:795-803.

29. Risser, G., Banihashemi, Z., and Davis, D. W. 1976. A proposed nomenclature of Fusarium oxysporum f. sp. melonis races and resistance genes in Cucumis melo. Phytopathology 66:1105-1106.

30. Schneider, R. W. 1984. Effects of nonpathogenic strains of Fusarium oxysporum on celery root infection by $F$. oxysporum $\mathrm{f}$. sp. apii and a novel use of the Lineweaver-Burk double reciprocal plot technique. Phytopathology 74:646-653.

31. Wattad, C., Freeman, S., Dinoor, A., and Prusky, D. 1995. A nonpathogenic mutant strain of Colletotrichum magna is deficient in pectate lyase activity. Mol. Plant-Microbe Interact. 8:621-626.

32. Wijesundera, R. L. C., Bailey, J. A., Byrde, R. J. W., and Fielding, A. H. 1989. Cell wall degrading enzymes of Colletotrichum lindemuthianum: Their role in the development of bean anthracnose. Physiol. Mol. Plant Pathol. 34:403-413.

33. Yamaguchi, K., Sano, T., Arita, M., and Takahashi, M. 1992. Biocontrol of Fusarium wilt of tomato and Verticillium wilt of eggplant by nonpathogenic Fusarium oxysporum MT0062. Ann. Phytopathol. Soc. Jpn. 58:188-194. 